\title{
Genomics at the evolving species boundary
}

Carlos A. Arias ${ }^{*}, 1,4$, Steven Van Belleghem ${ }^{*}, 1,2,3$ and W. Owen McMillan ${ }^{*}, 1$

${ }^{1}$ Smithsonian Tropical Research Institute, Apartado 0843-03092, Panamá, República de Panamá

2 Department of Zoology, University of Cambridge, Cambridge CB2 3EJ, United Kingdom

${ }^{3}$ Department of Biology and Center for Applied Tropical Ecology and Conservation, University of Puerto Rico, Rio Piedras, Puerto Rico

${ }^{4}$ Biology Program, Faculty of Natural Science and Mathematics. Universidad del Rosario. Carrera 24 \# 63c-69. Bogotá, 111221, Colombia.

* All authors contributed equally 


\begin{abstract}
Molecular studies on hybridization date back to Dobzhansky who compared chromosomal banding patterns to determine if interspecific gene flow occurred in nature [1]. Now, the advent of high-throughput sequencing provides increasingly fine insights into genomic differentiation between incipient taxa that are changing our view of adaptation and speciation and the links between the two. Empirical data from hybridizing taxa demonstrate highly heterogeneous patterns of genomic differentiation. Although underlining reasons for this heterogeneity are complex, studies of hybridizing taxa offers some of the best insights into the regions of the genome under divergent selection and the role these regions play in species boundaries. The challenge moving forward is to develop a better theoretical framework that fully leverages these powerful natural experiments.
\end{abstract}


Hybrid zones (HZs) are regions where genetically distinct populations meet, mate and produce offspring of mixed ancestry [2]. These zones are often narrow relative to the geographic distributions of the parental populations and are associated with steep clines in phenotypic and genetic variation [2-5]. HZs are typically classified along several axes, including geographic structure (mosaic versus clinal), historical origin (primary versus secondary contact), and the degree of reproductive isolation (unimodal versus bimodal). They are ubiquitous in animals and plants and create remarkable natural experiments for research into the nature of species and population differences.

There is a long history of hybridization research and the study of insect $\mathrm{HZs}$ has catalyzed debate about i) genomic architecture of adaptation and speciation [6], ii) the importance of hybridization in adaptation [ $\left.7^{*}\right]$, and iii) the role of natural selection in speciation $[8,9]$. Advancing technologies allow us to characterize differences across the genome with increasingly fine detail and make research on hybridizing taxa even more relevant today. Here, we highlight some recent research on insect HZs that demonstrate how genomic data is being integrated into traditional $\mathrm{HZ}$ research to increase our understanding of ecological and evolutionary processes. Moreover, we use this opportunity to stress some analytical limitations that need to be solved to take full advantage of genomic data and move $\mathrm{HZ}$ research forward. For more general recent reviews on hybridization and $\mathrm{HZs}$, see Harrison and Larson [7*] and Abbott, et al. [10].

Genomics across the speciation continuum: Genomic research on hybridizing taxa at various stages across the speciation continuum (Figure 1) extends decades of ecological studies providing rich empirical systems for addressing some of the most challenging questions in evolutionary and ecological research, including i) what is the architecture of complex adaptations [11-15]?, ii) how does natural selection and gene flow shape genome-wide patterns of divergence [16-18**-20*]?, and iii) how might species adapt to a rapidly changing world [21-24]? 
In HZs where hybridization is frequent (Stage 1 in Figure 1), differentiation is expected only around regions of the genome closely associated with functionally important variation. These regions contribute to isolation during the earliest stages of divergence and pinpointing their location, their number, and their effect size is important for our understanding of the architecture of adaptive variation and how this architecture either facilitates or impedes further divergence. In these zones, reduced gene flow at loci involved in (or closely linked to) the traits underlying divergent traits will be highly differentiated relative to regions of the genome not under selection (Figure 1B). High resolution divergence mapping or population association mapping can, therefore, be extremely useful in localizing genomic regions and genes involved in phenotypic differences, particularly when traits have a relatively simple genetic basis ([25**]; Box 1). Work on the genetic basis of mimetic wing color patterns of Heliconius butterflies provides a particularly compelling example of how genomic technologies can be used to identify functionally important variation. Many Heliconius species are composed of a number of highly divergent color pattern races. Color pattern races are often, but not always [26], distributed parapatrically and linked together by a patchwork of narrow $\mathrm{HZs}$ [13]. Classical crossing experiments have shown that much of the color variation across the genus is controlled by allelic variation at a handful of major effect loci [27]. Recently, association mapping across replicate $\mathrm{HZs}$ among divergent color pattern races has identified candidate genes and the functional regions around them that cause the striking variation in the vivid wing patterns that characterize the group [11,13,28-30] (Box 1).

Later stages in the speciation process (Stage 2 and 3, Figure 1) are characterized by a strong reduction in the frequency of hybrids because of a combination of ecological and reproductive differences. Taxa at these stages along the continuum provide a wealth of information about the relative importance of extrinsic and intrinsic forces in the earliest stages of speciation. Classic insect examples include scenarios in which ecological divergence (extrinsic) results in assortative mating and reduced hybridization as a 
byproduct. For instance, divergence in host-plant use in Timema walking sticks [31], fruit flies in the genus Rhagoletis [32] and the pea aphid Acyrthosiphon pisum [33] has shown to be a key factor resulting in assortative mating on host plants in the early stages of speciation. In other systems, such as the field crickets of the genus Gryllus [34] and Heliconius butterflies [35,36], divergence in mating cues, not directly related to mating location, are important. Additionally, hybrids often show intrinsic postzygotic isolation that reveals the importance of other processes, including different rates of evolution on the sex chromosome, in the origins of reproductive isolation $\left[37,38^{*}, 39\right]$. Study of $\mathrm{HZs}$ with these types of dynamics has profoundly shaped our understanding of how speciation occurs, including a renewed appreciation of the role of natural selection in the origins of new species and the recognition that certain genomic architectures facilitate speciation $[40,41]$.

The application of genomic technologies to the study of taxa where hybrids are rare permits insight into how genomes diverge during speciation and how a permeable species boundary can contribute to adaptation through a process called adaptive introgression (Box 2). Not surprisingly, patterns of genomic divergence among these taxa are far more complex than those observed between freely hybridizing taxa (Figure 1). Indeed, one of the strongest patterns emerging from genomic research is just how highly heterogeneous the patterns of genomic divergence are between hybridizing taxa. The heterogeneity reflects a complex interplay between natural and sexual selection, gene flow, demography, and recombination in divergence and admixture.

Unfortunately, differing combinations of evolutionary processes and modes of speciation can generate similar patterns of genomic divergence. For example, the evolution of reproductive barriers results in a genome wide reduction of gene flow, which should permit genome-wide differentiation. Similarly, even with moderate gene flow, selection at multiple loci involved in ecological adaptation may not only reduce effective gene flow at regions physically linked to these genes, but also across the entire genome, a process called genomic hitchhiking [42,43*]. This is particularly true when 
the traits under selection are influenced by a large number of interacting loci [44]. The rate at which genomes "congeal" into highly differentiated units can be rapid $[16,45]$ and disentangling the factors driving divergence is challenging (Box 3 and below). Of course, genome data are much more informative when combined with detailed ecological studies and coupled with other types of genetic, functional genetic, and experimental research $[46,47]$.

Despite the challenges with inferring process from pattern, genomic examination of highly differentiated taxa has demonstrated just how porous the species boundary actually is. As data accumulate, we are increasingly recognizing that species are often incompletely isolated and exchange genetic material for millions of years after they form. For example, recent studies suggest that up to $40 \%$ of the genome is exchanged between sympatric and ecologically divergent Heliconius species $[16,18]$. These data also include some of the most compelling examples of adaptive introgression (Box 2) between well-differentiated taxa, challenging the view that hybridization is always "unnatural and maladaptive" [48]. In this respect, a porous species boundary provides a source of new genetic variation and hybridization has been shown to have played an important role in the radiation of wing color patterns in Heliconius butterflies [49], as well as, the spread of insecticide resistance alleles and changes in ecological traits that influence the spread of human malaria in the Anopheles species complex [50,51**] (Box 2).

Moving forward: HZs will continue to provide exceptional experimental systems for combining genomic data with data on naturally occurring variation in form, function, and fitness. Genomic technologies will improve and, as they do, it is easy to imagine $\mathrm{HZ}$ studies where fine-scale descriptions of genomic variation from many individuals are layered onto one another and integrated with data on individual differences in morphology, ecology, and behavior. 
To take full advantage of these incredibly rich data sets requires the continued elaboration of theory and statistical methods that more fully leverage all the information contained within genomic data (Box 3). The summary statistics often used to quantify genetic differences and to identify genomic regions where gene flow is restricted or where admixture has occurred can be misleading and inferences drawn from them can be wrong. As an example, Cruickshank and Hahn [52*] recently reanalyzed several datasets that identified areas of elevated divergence, so-called "islands" of speciation or differentiation, in the genomes of hybridizing taxa. The "island" metaphor was first coined by Turner and colleagues [53] who, in one of the first population genomic studies, identified several highly divergent regions in genomes of hybridizing but ecological divergent strains of $A$. gambiae [53]. The metaphor has since become established in the lexicon of evolutionary biology [54], because the underlying logic is so compelling. In taxa that hybridize but maintain their distinctiveness, those regions that are resistant to gene flow are likely to harbor the variation responsible for differences in ecology, morphology and behavior. However, Cruickshank and Hahn [52*] found that islands disappeared when they used a measure of divergence that was insensitive to levels of within species variation. Moreover, in all cases, previously identified islands fell in regions of the genome with reduced diversity leading Cruickshank and Hahn to hypothesize that the observed patterns of differentiation were best explained by local selective sweeps, rather than restricted gene flow. These regions may still harbor functionally important variation, but not necessarily the variation underlying the differences that distinguish incipient forms. This is a somewhat subtle but important distinction.

The debate surrounding what forces, or combination of forces, generate the highly heterogeneous patterns of genomic differentiation observed among closely related taxa underscores the need to develop a more realistic theoretical framework of how genomes diverge under different evolutionary scenarios [55]. There is progress on this front [56**] (Box 3); however, most current methods do not incorporate recombination 
nor linkage disequilibrium (LD) into their models of divergence. The extent, distribution, and patterns of $L D$, in particular, potentially provide much finer understanding of the history of selection, admixture and population size changes. Fortunately, new theoretical frameworks are emerging that use recombination and LD and more fully leverage the information contained in genomic datasets. These new approaches allow the recovery of the history of admixture in natural populations (see $[57,58]$ ) and offer exciting new research possibilities. In some systems, it should be possible to contrast the recombination landscape, obtained from recombination maps of controlled crosses, with that observed in natural hybridizing populations to determine the relative importance of the recombination landscape and selection in admixture [59]. Indeed, as theory catches up with technology, we will be much better positioned to disentangle "the complex interplay between selection, gene flow, and recombination" [56] that occurs when genomes collide in HZs. This is absolutely essential if we are to understand the genomic architectures of the traits under divergent selection and their relative contribution in the speciation process. In this light, comparative genomic data from insect taxa from across the speciation continuum will almost certainly be at the forefront of our continued efforts to understand the buildup of genomic differences and to link them to the origins of novel variation, the evolution of reproductive isolation and the formation of new species. 


\section{Acknowledgements}

This work was supported by funding from the United States National Science

Foundation (DEB 1257689), the Smithsonian Tropical Research Institute, and the Smithsonian Institution's Competitive Grants program. 


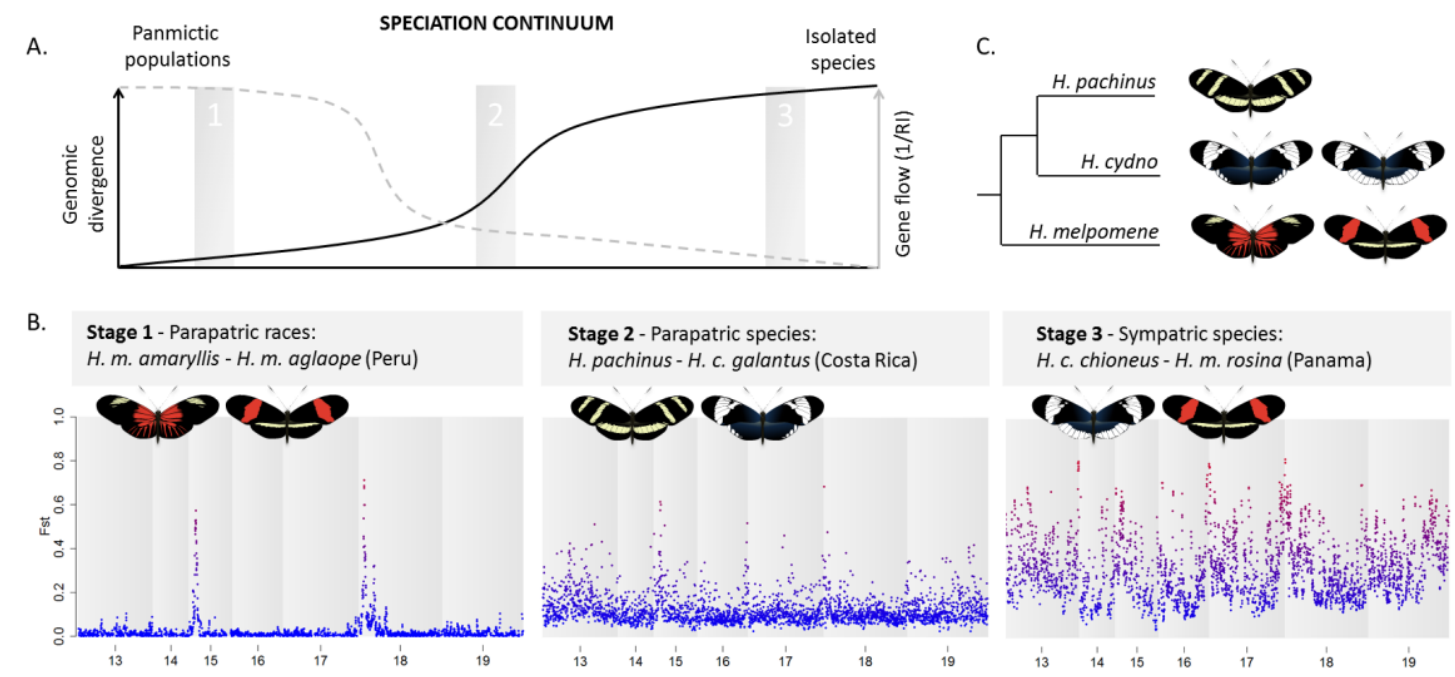

Figure 1: Hybrid zones provide windows into the genomic pattern of differentiation at different stages of the speciation process. (A.) The speciation process is generally argued to be a continuous process with the frequency of hybridization decreasing with the buildup of reproductive isolation (RI) and genetic distance. However, at the genomic level this process is not expected to be linear [43]. Rather, as genetic differentiation increases and RI evolves, gene flow strongly decreases on a genome wide scale resulting in rapid transitions along the speciation continuum. Identifying and analyzing these transitions helps elucidate the relative importance of the various intrinsic and extrinsic factors in speciation. As indicated in the figure, the correlation between the reduction in gene flow (represented by the dashed line) by the evolution of RI and the increase in genomic differentiation (represented by the solid line) may not be perfect due to genome wide reduction in gene flow resulting from selection on multiple loci. (B.) The pattern of differentiation (100 kb Fst windows) along the genome (chromosomes 13 to 19) of increasingly divergent Heliconius populations. The left panel shows differentiation between parapatric wing color pattern races which form HZs composed primarily of admixed individuals. The two peaks in the panel are associated with yellow (chromosome 15) and red (chromosome 18) color pattern variation. When genomic differences accumulate as the hybridization frequency decreases, it becomes increasingly difficult to infer associations between loci and traits (Stage 2 and 3). (C.) 
Phylogeny between the Heliconius species compared in the Fst plots. Data adapted from $[16,18]$. 


\section{Box 1: Population mapping in contact zones can help identify functionally important}

variation. Population association mapping refers to experiments conducted on natural populations in which relatedness among individuals is largely unknown [60]. In this respect, $\mathrm{HZs}$ provide powerful comparative opportunities to explore the genetic basis of adaptation and reproductive isolation and the links between them. The underlying logic is simple- these zones often represent many generations of hybridization and admixture between divergent taxa. This mixing creates recombinant genomes and different areas of the genome are expected to mix differently depending on a combination of intrinsic, extrinsic and random factors.

Several recent studies in insects have used population mapping and these studies serve to highlight the utility (and limitation) of this approach in natural populations. Population mapping between taxa with high levels of hybridization and few reproductive barriers (Stage 1 in the speciation continuum, see Figure 1B) can help pinpoint variation responsible for phenotypic differences, especially when combined with other evidence [47]. These zones are typically composed of highly admixed individuals, the practical result of which is that any associations between DNA sequence variants and a phenotype of interest are likely due to linkage with the functional variation that underlies phenotypic differences and not other phenomena such as population structure. In Heliconius butterflies, for example, population genomic analyses of HZs between divergent wing color pattern races identify a small genomic interval that likely underlies differences in red pattern variation [13] (Figure B1). This approximately $65 \mathrm{~kb}$ region shows a strong molecular signature of selection and contains numerous single nucleotide polymorphisms (SNPs) that are perfectly associated with phenotype. This divergent peak presumably contains a number of discrete regulatory regions of the transcription factor optix consistent with previous research that suggests that expression differences in optix underlies red pattern variation across the broader Heliconius wing color pattern radiation [11]. The striking divergence and genotype $x$ phenotype association around optix stands in marked contrast to the lack of differentiation in other regions of the genome (Figure B1). 
At the other extreme of the speciation continuum, population mapping of highly divergent taxa with strong ecological and reproductive differences can be informative about the genetic architecture of factors involved in adaptation and speciation. However, inferences drawn from the genomic dissection of these taxa are challenging, as reduced gene flow generates genome wide differentiation that obscures the association between genotype and phenotype [25**]. For a reasoned discussion of the power and limits of population mapping of divergent taxa see recent work by Gompert, et al. [61] on the genetic architecture of differences in female host plant preference and male genitalia in Lycaeides. As our ability to finely sample genomic variation and our theoretical understanding of the forces that shape this variation improve, population mapping of HZs promises increased understanding of the genetic basis of phenotypic variation and reproductive isolation. 


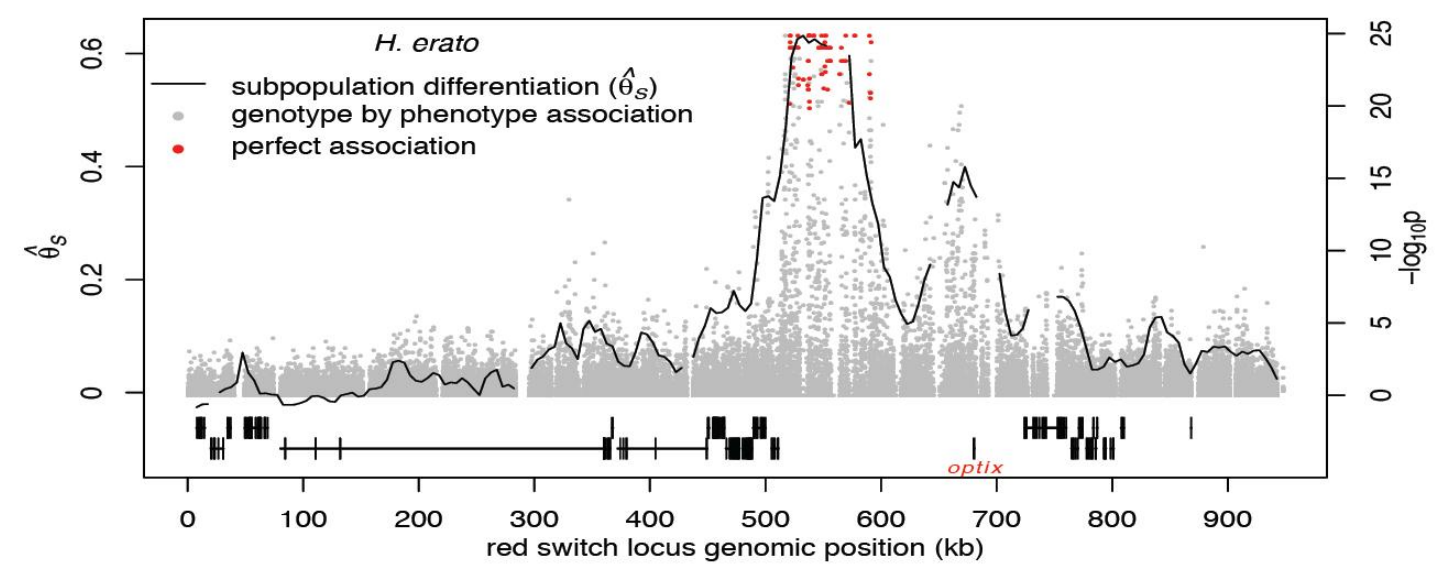

Figure B1: Sliding window genomic divergence between two major $H$. erato phenotypes ("postman" and "rayed") from three hybrid zones across a $1 \mathrm{Mb}$ genomic region that contains the red switch locus. There are two peaks of divergence, one centered on the transcription factor optix and another, much larger $65 \mathrm{~kb}$ peak, in a region downstream of optix. The $65 \mathrm{~kb}$ peak is in the center of a region containing no transcribed genes and is of particular interest as it also contains numerous SNPs (shown in red) perfectly associated with color phenotype (right hand axis). The two divergent peaks stand in marked contrast to the lack of differentiation at regions unlinked to color pattern, where average background genomic differences were effectively zero using a relative measure of between population differences (left hand axis). Figure modified from [13]. 
Box 2. Introgressive hybridization and its role in adaptation: Introgressive hybridization is the exchange of alleles between species through hybridization and subsequent gene flow. Introgressive hybridization has been recognized for over 60 years [62] but its relative importance in evolution remains contentious. Nonetheless, there is a growing recognition that hybridization is common and the advent of genomic technologies has demonstrated that species boundaries are extremely porous [7*]. As a creative force, introgressive hybridization can accelerate evolution by bringing together new adaptive combinations of alleles without the need to wait for mutations. Indeed, two recent studies of the Anopheles gambiae species complex emphasize an important role for introgressive hybridization in the diversification of the group. Several species in this complex are major vectors of human malaria and in the past few years there has been a rapid increase in genomic resources in the group, including the publication of 16 Anopheles genomes [63]. In one study, Fontaine et al. [51**] combined the new reference genomes with resequence data from many individuals across the range of the complex to document pervasive introgression among species (Figure B2). Their conclusions about the extent and importance of introgression rely on determining the correct historical branching order of the complex (i.e. the species tree). This is a nontrivial task in recently derived species because allele sharing among species could simply reflect the incomplete sorting of ancestral variation. Fontaine and colleagues went to great lengths to reconstruct the "true" relationships among species and to use this as the backdrop to identify introgressed genomic regions. In the Anopheles complex, phylogenetic relationships among species varied dramatically across different regions of the autosomes and between autosomes and X chromosome (Figure B2). They argued that the correct branching order of species was retained in genomic regions of the chromosomes that show the deepest divergence, which were particularly abundant on the $\mathrm{X}$ chromosome. Under this assumption, Fontaine et al. detected several major introgression events across the complex that appeared to play a role in adaptation. This included a large region that was shared among the three more distantly related anthropophilic species, $A$. gambiae, $A$. coluzzii, and $A$. arabiensis, that may have played 
an important role in the emergence of traits enhancing malaria transmission to humans. In addition, they identified another introgression on chromosome 2 (2La) from the ancestral $A$. gambiae species into $A$. arabiensis that has been shown to confer resistance to desiccating environments (Figure B2). In another study, Clarkson et. al. [50] examined the rise of an insecticide-resistance mutation in the $\mathrm{M}$ and $\mathrm{S}$ forms of $A$. gambiae using whole genome resequence data. Despite strong isolation between the forms, the new mutation has spread from the $\mathrm{S}$ to $\mathrm{M}$ form and rapidly increased in frequency consistent with strong natural selection. By sequencing the full genomes of 15 wild-caught individuals, the group showed that the introgessed region was massive (about $3 \mathrm{Mb}$ ) and completely removed a very large peak of divergence between the two forms. Nonetheless, this introgression event did not obviously reduce the strong reproductive barriers between the two forms, recently characterized as distinct species [64]. Taken together, these two studies demonstrate that introgressive hybridization can provide the raw material for evolution without necessarily eroding the differences in ecology and mating behavior that characterize incipient species. 


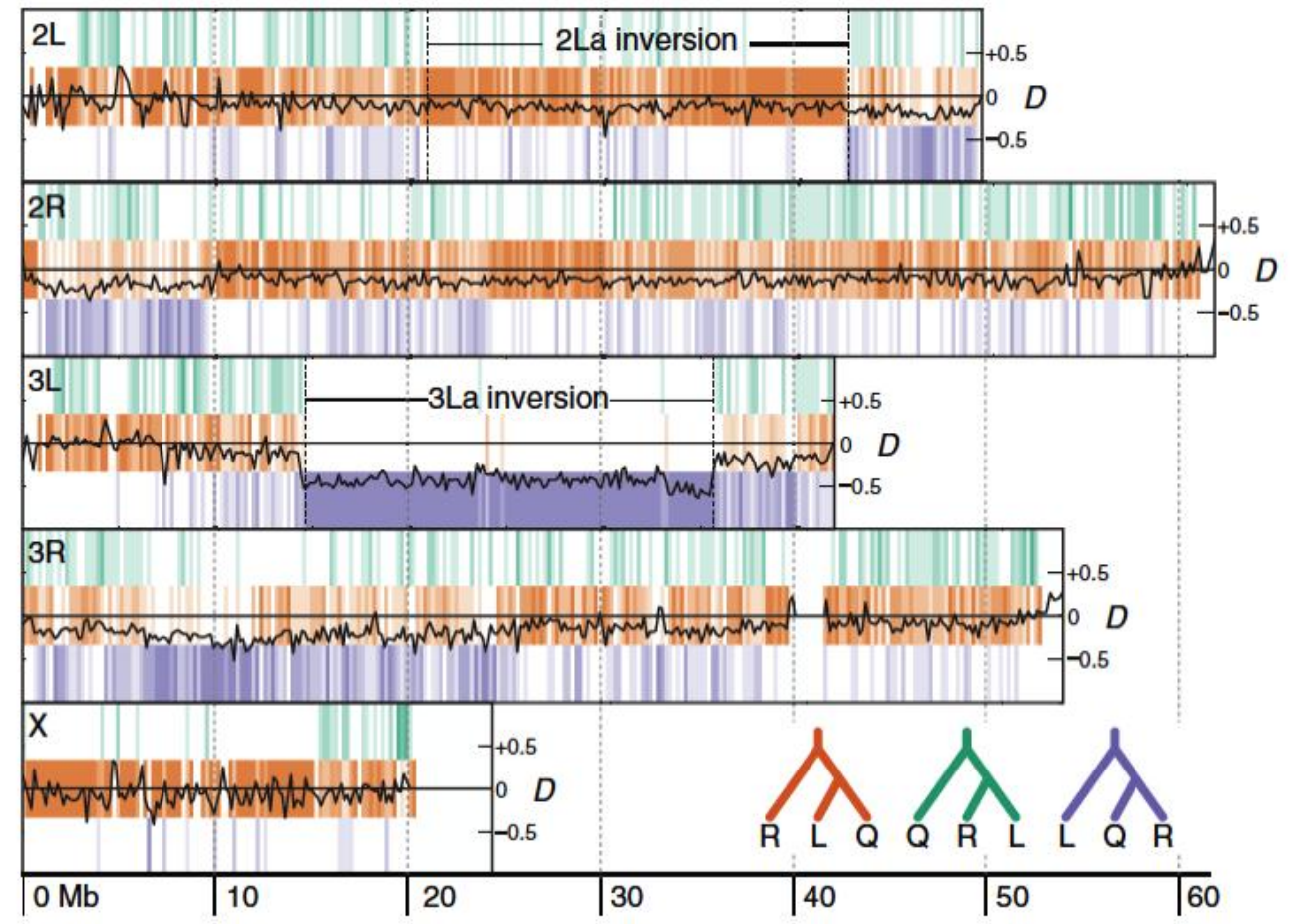

Figure B2: Introgression within the Anopheles gambiae complex. Most likely phylogenetic relationships among $A$. quadriannulatus (Q), A. melas (L) and A. merus (R) and Patterson's D based on sliding window calculations across 4 autosomal chromosomal arms and the $\mathrm{X}$ chromosome. The orange phylogeny is thought to reflect the true branching order of the three species. Comparing this expected phylogeny to the best support phylogeny generated at different gene regions indicates a large amount of phylogenetic discordance across the genome. This is particularly true when comparing gene trees generated from the $X$ chromosome, which generally reflect the true tree, and those generated from autosome regions, which reflect the species tree less than $50 \%$ of the time. At least some of this discordance can be explained by introgressive hybridization. As an example, there is a large block in 3L where Patterson's $D$ is consistently negative indicating an excess of shared derived alleles and strongly suggestive of introgression from $A$. merus (R) to $A$. quadriannulatus (Q). Figure modified from $\left[51^{* *}\right]$. 
BOX 3. Analytical tools used in genomic research. High throughput sequencing strategies will continue to improve and the current challenge is to develop analytical methods that make use of all the information in these rich, albeit large and complex, datasets. To date, most studies of hybridizing taxa have relied on inferring aspects of the history of divergence and gene flow from relatively simple summary statistics or very basic evolutionary models. For instance, many studies have identified highly divergent genomic regions using the Fst statistic, which measures the normalized difference in allele frequency between populations [65]. Alternatively, studies have estimated the degree of admixture between taxa using Patterson's D statistic [66]. This statistic and variants of it [67] measure the excess of shared derived alleles in regions across the genome as way to identify introgressed loci. Within the context of HZs, classic "cline theory" [3] can be used to identify genomic regions that contribute to species boundaries and/or local adaptation. In its simplest form, cline theory models allele frequency differences between hybridizing taxa as a function of selection and dispersal. Thus, alleles that show abrupt changes in frequency across a hybrid zone may be important for the ecological, morphological, and behavioral differences that characterize hybridizing taxa. Recently, Buerkle and colleagues have applied cline theory at the genomic level [68-70]. Their method of genomic clines relies on generating the expected distribution of admixture at neutral loci in two populations coming into secondary contact. Loci that deviate from the neutral expectations are likely experiencing selection and different forms of selection can, in theory, be distinguished.

The above strategies for analyzing genomic data enriched our understanding of genomic divergence and focused debate around what these patterns actually mean. This in turn has stimulated efforts to develop a more robust theoretical framework for analyzing genomic data. Statistical approaches are beginning to emerge that measure the likelihood of particular patterns of divergence under specific evolutionary models. These models make fuller use of genome data and concomitantly allow researchers to estimate specific parameters from the data. For instance, the observed allele frequency spectrum (AFS), which summarizes the joint distribution of allele frequencies across 
diallelic variants in single or multiple populations, can be compared to the AFS under a specific demographic model to distinguish among competing modes of speciation $[71,72]$. Alternatively, the pattern of branching events in allelic genealogies from multiple gene trees can be examined and compared to those expected under competing evolutionary scenarios using coalescent theory $[73,74]$. These approaches assume that SNPS or gene regions used in the analysis are independently segregating. Furthermore, coalescent-based approaches assume no recombination within genomic regions being analyzed. More recent mathematical modeling approaches are beginning to make use of the patterns of linkage disequilibrium (LD) and haplotype information across the genome [58]. When coupled with an understanding of variation in recombination rate across the genome, these approaches will more effectively use information within genomic datasets. Presently, the broad application of these methods is restricted because they require both a good reference genome and phased diploid genomic data and are computationally intensive even when comparing small numbers of samples [75]. For an exceptional overview of state-of-the-science analytical methods and the challenges of making full use of genomic data see [56**]. 


\section{Glossary:}

Adaptive introgression: Gene flow of advantageous alleles from one species into the gene pool of another by repeated backcrossing.

Admixture: The exchange of genetic material between populations or closely related species resulting in chromosomal regions of mixed ancestry.

Allele Frequency Spectrum: Matrix containing the number of diallelic genetic polymorphisms (in a whole genome or genomic region) in which the derived or minor allele was found in the corresponding number of samples from each population. The dimension of the matrix is equal to the number of populations.

Bimodal hybrid zone: $\mathrm{HZ}$ consisting largely of individuals resembling the parental forms with low frequencies of intermediate genotypes and/or phenotypes.

Clinal hybrid zone: $\mathrm{HZs}$ that show a gradual transition from one character state to another, typically associated with steep clines in phenotypic and genetic variation, and often associated with distinct environmental transitions.

Coalescent theory: The population genetic theory that describes the expected distribution of time to common ancestry of a set of alleles drawn from a population or several populations under a given set of demographic conditions.

Divergence Mapping: Using scans of genetic markers to identify ones that show allele frequency differences that are higher than expected by random chance. Similar to population association mapping, this strategy is based on the premise that the marker(s) showing elevated rates of divergence are linked to functionally important variation. Importantly, a number of approaches have been developed that incorporate underlying neutral population genetic structure.

Extrinsic factors promoting genomic differentiation: Barriers to gene flow that arise when hybrids experience lower fitness in a particular environment.

Fst (Wright's fixation index): A measure of population differentiation. It is calculated as the fraction of the total genetic variation that is distributed among subpopulations.

Gene tree: A bifurcating tree that represent the ancestral relationships of homologous loci (more correctly haplotypes) sampled from closely related species. The topology may or may not reflect the topology of the species tree depending on the history of selection and gene flow.

Genomic architecture: Refers to the number, location, and effect size of loci underlying a phenotypic trait. 
Genomic clines: Mathematical functions that describe the probability of locus-specific ancestry along a gradient in genome-wide admixture or hybrid index. Hybrid index is defined as the proportion of an admixed individual's genome inherited from one of two parental populations.

Intrinsic factors promoting genomic differentiation: Barriers to gene flow driven by incompatibilities in hybrids independent of the environment.

Linkage disequilibrium (LD): The nonrandom association of alleles at different loci. The level of LD is determined by a number of factors including physical linkage, population structure, and selection. LD is broken down by recombination.

Mosaic hybrid zones: $\mathrm{HZs}$ that show no regular transition from one parental taxon to the other. Rather parental forms associate with particular habitat mosaics, where one parental taxa is associated with one habitat type and the other with another habitat type and hybridization occurs across patch boundaries.

Patterson's $D$ statistic: A statistic that uses the distribution of shared derived alleles between taxa to identify interspecific gene flow. The statistic is based on a simple premise: when considering an outgroup, alleles can be either ancestral ("A) or derived ("B"). When considering four taxa, derived alleles can be shared among taxa resulting in patterns termed "ABBA" and "BABA". An excess of ABBA or BABA patterns is indicative of gene flow between two of the taxa (see $[66,67])$.

Population Association Mapping: Uses the statistical association between genetic markers and particular phenotypes obtained from a collection of population samples to identify regions of the genome that may be responsible for phenotypic variation. The strategy is based on the premise that the marker(s) associated with a particular phenotype will be in close proximity to the causal locus or loci determining a phenotype.

Primary contact: When divergence takes place in situ between adjacent populations of a previously homogeneous species.

Secondary contact: Contact between two diverged lineages that were previously geographically isolated.

Speciation continuum: A re-envisioning of Darwin's view of speciation where there is a continuous sequence of genetically-based changes that occur as two lineages diverge from one another on the pathway to complete reproductive isolation [76].

Species tree: A bifurcating tree that reflects the true history of speciation.

Unimodal hybrid zone: $\mathrm{HZ}$ with high frequencies of intermediate genotypes and/or phenotypes. 


\section{References}

1. Dobzhansky T: Is there gene exchange between Drosophila pseudoobsura and Drosophila persimilis in their natural habitats? The American Naturalist 1973, 107:312-314.

2. Barton N, Hewitt G: Analysis of hybrid zones. Annual Review of Ecology and Systematics 1985, 16:113-148.

3. Barton N: The dynamics of hybrid zones. Heredity 1979, 43:341-359.

4. Arias CF, Rosales C, Salazar C, Castaño J, Bermingham E, Linares M, McMillan WO: Sharp genetic discontinuity across a unimodal Heliconius hybrid zone. Molecular Ecology 2012, 21:5778-5794.

5. Ross C, Harrison R: A fine-scale spatial analysis of the mosaic hybrid zone between Gryllus firmus and Gryllus pennsylvanicus. Evolution 2002, 56:2296-2312.

6. Barton NH: Genetic linkage and natural selection. Philosophical Transactions of the Royal Society of London B: Biological Sciences 2010, 365:2559-2569.

7. Harrison RG, Larson EL: Hybridization, introgression, and the nature of species boundaries. Journal of Heredity 2014, 105:795-809.

* A recent extensive review on gene exchange along the genome between hybridizing taxa and its implications for defining species boundaries. A comprehensive overview is given of studies comparing genome wide patterns of differentiation, as well as, studies investigating patterns of introgression.

8. Schluter D: Evidence for ecological speciation and its alternative. Science 2009, 323:737-741.

9. Soria-Carrasco V, Gompert Z, Comeault AA, Farkas TE, Parchman TL, Johnston JS, Buerkle CA, Feder JL, Bast J, Schwander T, et al.: Stick insect genomes reveal natural selection's role in parallel speciation. Science 2014, 344:738-742.

10. Abbott R, Albach D, Ansell S, Arntzen JW, Baird SJE, Bierne N, Boughman J, Brelsford A, Buerkle CA, Buggs R, et al.: Hybridization and speciation. Journal of Evolutionary Biology 2013, 26:229-246.

11. Reed RD, Papa R, Martin A, Hines HM, Counterman BA, Pardo-Diaz C, Jiggins CD, Chamberlain NL, Kronforst MR, Chen $\mathrm{R}$, et al.: Optix drives the repeated convergent evolution of butterfly wing pattern mimicry. Science 2011, 333:1137-1141.

12. Nadeau NJ, Whibley A, Jones RT, Davey JW, Dasmahapatra KK, Baxter SW, Quail MA, Joron $\mathrm{M}$, ffrench-Constant $\mathrm{RH}$, Blaxter $\mathrm{ML}$, et al.: Genomic islands of divergence in hybridizing Heliconius butterflies identified by large-scale targeted sequencing. Philosophical Transactions of the Royal Society B: Biological Sciences 2012, 367:343-353.

13. Supple MA, Hines HM, Dasmahapatra KK, Lewis JJ, Nielsen DM, Lavoie C, Ray DA, Salazar C, McMillan WO, Counterman BA: Genomic architecture of adaptive color pattern divergence and convergence in Heliconius butterflies. Genome Research 2013, 23:12481257.

14. Gompert Z, Lucas LK, Nice CC, Buerkle CA: Genome divergence and the genetic architecture of barriers to gene flow between lycaeides idas and I. melissa. Evolution 2013, 67:24982514.

15. Zhang W, Kunte K, Kronforst MR: Genome-wide characterization of adaptation and speciation in tiger swallowtail butterflies using de novo transcriptome assemblies. Genome Biology and Evolution 2013, 5:1233-1245. 
16. Kronforst Marcus R, Hansen Matthew EB, Crawford Nicholas G, Gallant Jason R, Zhang W, Kulathinal Rob J, Kapan Durrell D, Mullen Sean P: Hybridization reveals the evolving genomic architecture of speciation. Cell Reports 2013.

17. Larson EL, White TA, Ross CL, Harrison RG: Gene flow and the maintenance of species boundaries. Molecular Ecology 2014, 23:1668-1678.

18. Martin SH, Dasmahapatra KK, Nadeau NJ, Salazar C, Walters JR, Simpson F, Blaxter M, Manica A, Mallet J, Jiggins CD: Genome-wide evidence for speciation with gene flow in Heliconius butterflies. Genome Research 2013, 23:1817-1828.

** The paper presents an interesting example of in depth analysis of whole genome sequences by focusing on the distribution of genealogical relationships along the genome and patterns of linkage disequilibrium. By sequencing whole genomes of 31 individuals from multiple Heliconius populations and species they demonstrate that a substantial fraction (up to $40 \%$ of $100 \mathrm{~kb}$ genomic windows) of the genome has been shared by ongoing hybridization between sympatric species.

19. Noor MAF, Garfield DA, Schaeffer SW, Machado CA: Divergence between the Drosophila

pseudoobscura and $D$. persimilis genome sequences in relation to chromosomal

inversions. Genetics 2007, 177:1417-1428.

20. Gompert Z, Lucas LK, Buerkle CA, Forister ML, Fordyce JA, Nice CC: Admixture and the organization of genetic diversity in a butterfly species complex revealed through common and rare genetic variants. Molecular Ecology 2014, 23:4555-4573.

* The manuscript reports the effect of admixture and spatial isolation on the organization of genome-wide variation and ancestry in populations of the Lycaeides butterfly. Interestingly, different genomic variants (rare and common) present distinctive evolutionary histories that can be used to elucidate past demographic events. The study is also noteworthy because of its detailed discussion of the challenges of identifying functionally important variation using genome scan data of hybridizing populations.

21. Chunco AJ: Hybridization in a warmer world. Ecology and Evolution 2014, 4:2019-2031.

22. Mallet J, Wynne IR, Thomas CD: Hybridisation and climate change: brown Argus butterflies in Britain (Polyommatus subgenus Aricia). Insect Conservation and Diversity 2010.

23. Scriber J, Elliot B, Maher E, McGuire M, Niblack M: Adaptations to "thermal time" constraints in Papilio: latitudinal and local size clines differ in response to regional climate change. Insects 2014, 5:199.

24. Taylor SA, Larson EL, Harrison RG: Hybrid zones: windows on climate change. Trends in Ecology \& Evolution 2015, 30:398-406.

25. Crawford JE, Nielsen R: Detecting adaptive trait loci in nonmodel systems: divergence or admixture mapping? Molecular Ecology 2013, 22:6131-6148.

** This study combines an excellent historical background on strategies for identifying adaptive trait loci with simulations that help guide the design of genome-wide studies of adaptation in natural populations. Not unexpectedly, the power to identify adaptive trait loci decreases with increasing levels of background population differences. Their simulations modeled a very simple genetic architecture (a single major effect locus) and a restricted set of demographic conditions but the authors provide a nice discussion about how more complex scenarios will affect the ability of genomic data to identify functionally important variation in natural populations. 
26. Joron M, Mallet J: Diversity in mimicry: paradox or paradigm? Trends Ecol. Evol. 1998, 13:461-466.

27. Papa R, Kapan DD, Counterman BA, Maldonado K, Lindstrom DP, Reed RD, Nijhout HF, Hrbek T, McMillan WO: Multi-allelic major effect genes interact with minor effect QTLs to control adaptive color pattern variation in Heliconius erato. PLOS ONE 2013, 8:e57033.

28. Martin A, Papa R, Nadeau NJ, Hill RI, Counterman BA, Halder G, Jiggins CD, Kronforst MR, Long $A D$, McMillan WO, et al.: Diversification of complex butterfly wing patterns by repeated regulatory evolution of a Wnt ligand. Proceedings of the National Academy of Sciences 2012, 109:12632-12637.

29. Nadeau N, Pardo-Diaz C, Whibley A, Supple MA, Wallbank R, Wu GC, Maroja L, Ferguson L, Hines $\mathrm{H}$, Salazar $\mathrm{C}$, et al.: The origins of a novel butterfly wing patterning gene from within a family of conserved cell cycle regulators. bioRxiv 2015.

30. Nadeau N, Ruiz M, Salazar P, Counterman B, Medina JA, Ortiz-Zuazaga H, Morrison A, McMillan WO, Jiggins CD, Papa R: Population genomics of parallel hybrid zones in the mimetic butterflies, H. melpomene and H. erato. Genome Research 2014, 24:13161333.

31. Nosil P, Crespi B, Sandoval C: Host-plant adaptation drives parallel evolution of reproductive isolation. Nature 2002, 417:440-443.

32. Filchak KE, Roethele JB, Feder JL: Natural selection and sympatric divergence in the apple maggot Rhagoletis pomonella. Nature 2000, 407:739-742.

33. Hawthorne DJ, Via S: Genetic linkage of ecological specialization and reproductive isolation in pea aphids. Nature 2001, 412:904-907.

34. Maroja L, Andrés J, Walters J, Harrison R: Multiple barriers to gene exchange in a field cricket hybrid zone. Biological Journal of the Linnean Society 2009, 97:390-402.

35. McMillan W, Jiggins $C$, Mallet J: What initiates speciation in passion vine butterflies? Proceedings of the National Academy of Sciences, USA 1997, 94:8628-8633.

36. Jiggins C, Naisbit R, Coe R, Mallet J: Reproductive isolation caused by colour pattern mimicry. Nature 2001, 411:302-305.

37. Coyne J, Orr H: Patterns of speciation in Drosophila revisited. Evolution 1997, 51:295-303.

38. Garrigan D, Kingan SB, Geneva AJ, Vedanayagam JP, Presgraves DC: Genome diversity and divergence in Drosophila mauritiana: multiple signatures of faster $\mathrm{X}$ evolution. Genome Biology and Evolution 2014, 6:2444-2458.

* In depth analysis of population genomic data of D. mauritiana, showing that, relative to the autosomes, the $X$ chromosome has reduced nucleotide diversity but elevated nucleotide divergence, an excess of recurrent adaptive evolution at its protein-coding genes and an excess of recent, strong selective sweeps. These findings provide a likely explanation for the fast evolution of $X$ linked hybrid sterility and its large effect in speciation.

39. Presgraves D: Patterns of postzygotic isolation in Lepidoptera. Evolution 2002, 56:11681183.

40. Servedio MR, Doorn GSV, Kopp M, Frame AM, Nosil P: Magic traits in speciation: 'magic' but not rare? Trends in Ecology \& Evolution 2011, 26:389-397.

41. Nosil P, Feder JL: Genomic divergence during speciation: causes and consequences. Philosophical Transactions of the Royal Society B: Biological Sciences 2012, 367:332-342. 
42. Flaxman SM, Feder JL, Nosil P: Spatially explicit models of divergence and genome hitchhiking. Journal of Evolutionary Biology 2012, 25:2633-2650.

43. Feder JL, Nosil P, Wacholder AC, Egan SP, Berlocher SH, Flaxman SM: Genome-wide congealing and rapid transitions across the speciation continuum during speciation with gene flow. Journal of Heredity 2014, 105:810-820.

* This manuscript reviews an emergent process of genomic divergence, called genome-wide congealing (GWC), which involves the combined effect of multiple genes contributing to genome wide reduction in gene flow. They assess conditions that contribute to GWC, depict how to empirically test for such a process and present a putative empirical example in Rhagoletis fruit flies.

44. Flaxman SM, Feder JL, Nosil P: Genetic hitchhiking and the dynamic buildup of genomic

divergence during speciation with gene flow. Evolution 2013, 67:2577-2591.

45. Egan SP, Ragland GJ, Assour L, Powell THQ, Hood GR, Emrich S, Nosil P, Feder JL:

Experimental evidence of genome-wide impact of ecological selection during early stages of speciation-with-gene-flow. Ecology Letters 2015, 18:817-825.

46. Barrett RDH, Hoekstra HE: Molecular spandrels: tests of adaptation at the genetic level. Nature Review Genetics 2011, 12:767-780.

47. Stinchcombe JR, Hoekstra HE: Combining population genomics and quantitative genetics:

finding the genes underlying ecologically important traits. Heredity 2008, 100:158-170.

48. Mallet J: Hybridization as an invasion of the genome. Trends in Ecology and Evolution 2005, 20:229-237.

49. The Heliconius genome consortium: Butterfly genome reveals promiscuous exchange of mimicry adaptations among species. Nature 2012, 487:94-98.

50. Clarkson CS, Weetman D, Essandoh J, Yawson AE, Maslen G, Manske M, Field SG, Webster $M$, Antão T, Maclnnis B, et al.: Adaptive introgression between Anopheles sibling species eliminates a major genomic island but not reproductive isolation. Nature Communication 2014, 5: 4248-4258.

51. Fontaine MC, Pease JB, Steele A, Waterhouse RM, Neafsey DE, Sharakhov IV, Jiang X, Hall $A B$, Catteruccia $F$, Kakani $E$, et al.: Extensive introgression in a malaria vector species complex revealed by phylogenomics. Science 2015, 347:258524.

** The manuscript presents a compelling case of extensive introgression in the Anopheles gambiae complex. By taking advantage of the vast genomic resources available for this group of malaria vector mosquitoes, the authors first reconstruct the genealogical relationships of the A. gambiae complex and use this phylogeny as a framework to detect several major introgression events across the species complex that appear to have played a role in adaptation.

52. Cruickshank TE, Hahn MW: Reanalysis suggests that genomic islands of speciation are due to reduced diversity, not reduced gene flow. Molecular Ecology 2014, 23:3133-3157.

* A thoughtful paper that discusses the reasons for the highly heterogeneous patterns of genomic divergence that have been observed in recently derived species or forms. The authors challenge the conclusion that sharp peaks of differentiation observed in a diverse array of genomic datasets are the result of reduced gene flow. Instead, they suggest that these patterns are more likely driven by recent selective sweeps. 
53. Turner TL, Hahn MW, Nuzhdin SV: Genomic islands of speciation in Anopheles gambiae. PLoS Biol 2005, 3:e285.

54. Pennisi E: Disputed islands. Science 2014, 345:611-613.

55. Feder JL, Egan SP, Nosil P: The genomics of speciation-with-gene-flow. Trends in Genetics 2012, 28:342-350.

56. Sousa V, Hey J: Understanding the origin of species with genome-scale data: modelling gene flow. Nature Reviews Genetics 2013, 14:404-414.

** Comprehensive overview and discussion on the application of models and methods accommodated to genomic data and their suitability to solve evolutionary questions about the role of gene exchange in species formation.

57. Hellenthal G, Busby GBJ, Band G, Wilson JF, Capelli C, Falush D, Myers S: A genetic atlas of human admixture history. Science 2014, 343:747-751.

58. Leslie S, Winney B, Hellenthal G, Davison D, Boumertit A, Day T, Hutnik K, Royrvik EC, Cunliffe $B$, Welcome Trust Case Control Consortium, et al.: The fine-scale genetic structure of the British population. Nature 2015, 519:309-314.

59. Janoušek V, Munclinger P, Wang L, Teeter KC, Tucker PK: Functional organization of the genome may shape the species boundary in the house mouse. Molecular Biology and Evolution 2015, 32:1208-1220.

60. Myles S, Peiffer J, Brown PJ, Ersoz ES, Zhang Z, Costich DE, Buckler ES: Association mapping: critical considerations shift from genotyping to experimental design. The Plant Cell 2009, 21:2194-2202.

61. Gompert Z, Lucas LK, Nice CC, Fordyce JA, Alex Buerkle C, Forister ML: Geographically multifarious phenotypic divergence during speciation. Ecology and Evolution 2013, 3:595-613.

62. Anderson E: Introgressive hybridization. New York: Wiley \& Sons; 1949.

63. Neafsey DE, Waterhouse RM, Abai MR, Aganezov SS, Alekseyev MA, Allen JE, Amon J, Arcà $B$, Arensburger $P$, Artemov $G$, et al.: Highly evolvable malaria vectors: the genomes of 16 Anopheles mosquitoes. Science 2015, 347:1258522.

64. Coetzee M, Hunt RH, Wilkerson R, Torre AD, Coulibaly MB, Besansky NJ: Anopheles coluzzii and Anopheles amharicus, new members of the Anopheles gambiae complex. Zootaxa 2013, 3619:246-274.

65. Holsinger KE, Weir BS: Genetics in geographically structured populations: defining, estimating and interpreting FST. Nature Review Genetics 2009, 10:639-650.

66. Durand EY, Patterson N, Reich D, Slatkin M: Testing for ancient admixture between closely related populations. Molecular Biology and Evolution 2011, 28: 2239-2252.

67. Martin SH, Davey JW, Jiggins CD: Evaluating the use of ABBA-BABA statistics to locate introgressed loci. Molecular Biology and Evolution 2015, 32:244-257.

68. Buerkle CA: Maximum-likelihood estimation of a hybrid index based on molecular markers. Molecular Ecology Notes 2005, 5:684-687.

69. Gompert Z, Buerkle CA: A powerful regression-based method for admixture mapping of isolation across the genome of hybrids. Molecular Ecology 2009, 18:1207-1224.

70. Gompert Z, Buerkle CA: Analyses of genetic ancestry enable key insights for molecular ecology. Molecular Ecology 2013, 22:5278-5294. 
71. Excoffier L, Dupanloup I, Huerta-Sánchez E, Sousa VC, Foll M: Robust demographic inference from genomic and SNP data. PLoS Genetics 2013, 9:e1003905.

72. Gutenkunst RN, Hernandez RD, Williamson SH, Bustamante CD: Inferring the joint demographic history of multiple populations from multidimensional SNP frequency data. PLoS Genetics 2009, 5:e1000695.

73. Lohse K, Barton NH, Melika G, Stone GN: A likelihood-based comparison of population histories in a parasitoid guild. Molecular Ecology 2012, 21:4605-4617.

74. Wang Y, Hey J: Estimating divergence parameters with small samples from a large number of loci. Genetics 2010, 184:363-379.

75. Schiffels S, Durbin R: Inferring human population size and separation history from multiple genome sequences. Nature Genetics 2014, 46:919-925.

76. Shaw KL, Mullen SP: Speciation continuum. Journal of Heredity 2014, 105:741-742. 